Techniques \& Culture

43-44| 2004

Mythes. L'origine des manières de faire

\title{
Les techniques dans la pensée narrative
}

\section{François Sigaut}

\section{OpenEdition}

Journals

Édition électronique

URL : https://journals.openedition.org/tc/1241

DOI : $10.4000 /$ tc. 1241

ISSN : 1952-420X

\section{Éditeur}

Éditions de l'EHESS

\section{Édition imprimée}

Date de publication : 1 décembre 2004

ISSN : 0248-6016

\section{Référence électronique}

François Sigaut, "Les techniques dans la pensée narrative », Techniques \& Culture [En ligne], 43-44 2004, mis en ligne le 15 avril 2007, consulté le 29 septembre 2022. URL : http://

journals.openedition.org/tc/1241; DOI : https://doi.org/10.4000/tc.1241

Ce document a été généré automatiquement le 29 septembre 2022.

Tous droits réservés 


\title{
Les techniques dans la pensée narrative
}

\author{
François Sigaut
}

La mythologie

1 Toutes les sociétés et toutes les langues n'ont pas l'équivalent du concept que nous exprimons à l'aide des mots art et technique. Dans notre propre société, d'ailleurs, l'acception de ces termes est loin d'être uniforme. Mais quoi qu'il en soit, certaines techniques ont dans la vie humaine un rôle si important, et si évidemment important, qu'aucune société n'a pu l'ignorer tout à fait. C'est par certaines techniques -l'usage et la production du feu, la cuisine, l'emploi d'outils, le vêtement, etc.- que les hommes se distinguent des autres animaux. D'autres techniques, ou les mêmes mais considérées dans leurs variations -le tissage, la céramique, la métallurgie... -distinguent les groupes humains les uns des autres, en particulier «nous», les "vrais» hommes, raisonnables et civilisés, et " eux », les étranges étrangers, les barbares, les sauvages. Les techniques ne sont évidemment pas les seules à jouer ce double rôle distinctif : le langage et les mœurs le jouent aussi, dans les domaines les plus divers. Dans cet ensemble, chaque groupe social choisit d'insister sur ce qui l'arrange le mieux, sans pouvoir ni vouloir réserver aux techniques un traitement à part; ce qu'encore une fois, l'absence ordinaire d'un concept de technique ne permettrait pas.

2 Mais si la technique n'y est pas, les techniques, elles, sont partout. Et il faut bien les « expliquer ». Il faut rendre compte du feu, de la cuisson des aliments, du vêtement ou de la parure, de l'architecture, de l'agriculture et de l'élevage, de la navigation, de la forge... Tantôt il s'agira d'un don gracieux d'une divinité, tantôt au contraire, d'un vol dont le coupable sera dûment puni, et tous ses descendants avec lui. À l'opposé, c'est parfois le monde et l'homme qui sont expliqués par les techniques des dieux. Ici, le monde est un tissu. Là, les dieux fabriquent les montagnes en barattant la mer de lait. Et dans la Genèse, l'homme est pétri d'argile... Bien sûr, tous ces mythes sont dénués de sens si on les sépare de la société qui les a produits. C'est pour cela qu'il est tout à fait vain d'espérer tirer quelque chose d'une simple compilation. Et c'est aussi pour cela qu'on rêve d'une mythologie comparée à la Lévi-Strauss, mais qui se donnerait pour 
tâche de dégager les éléments de réflexion technologique si abondants dans les mythes, de déterminer quelle place ils y occupent, d'analyser leurs différences et leurs transformations.

Il existe des recherches qui vont dans ce sens, comme la passionnante lecture économique (qui est aussi largement technologique) des mythes de la Méditerranée antique que nous propose Morris Silver dans Taking Ancient Mythology Economically (1992). Nous y apprenons à reconnaître dans les prêtresses vierges des gardiennes du trésor, et dans les chiens des agents commerciaux. Les artisans itinérants sont, les uns borgnes, les autres boiteux, à cause du caractère tortueux de leurs circuits. Le démembrement et la résurrection du dieu Osiris font référence, non seulement au cycle des céréales, battage et semis, mais plus précisément justifient la dîme versée au temple, qui en contrepartie fournira la semence. Une autre justification de la dîme est le récit des amours de Demeter et de Jason "dans une jachère trois fois labourée " (sans contresens sur le terme jachère), d'où naîtra le dieu de la richesse, Ploutos. Quant à la lyre, une invention attribuée à Hermès, elle symbolise la corvée, parce que les chants et la musique étaient l'accompagnement nécessaire des travaux collectifs... Il ne faut peut-être pas ajouter la même foi à toutes les interprétations de M. Silver. Mais elles donnent toutes à penser, et l'on ne peut qu'adhérer d'enthousiasme à sa conclusion selon laquelle « les Anciens s'intéressaient beaucoup plus à l'économie [et j'ajouterai aux techniques] que les disciples de Karl Polanyi et de Moses Finley ne nous le donnent à croire ».

4 Mais le monde méditerranéen est un cas très particulier, tant par sa richesse matérielle que par le nombre et la diversité de ses mythes. En attendant de disposer d'études comparables à celle de M. Silver pour d'autres aires culturelles et d'autres époques en nombre suffisant, je me bornerai à évoquer quelques personnages bien connus qui me paraissent caractéristiques de la présence d'une réflexion sur les techniques dans le mythe et dans la fiction: le trickster et deux de ses avatars modernes, Gribouille et le savant fou; Faust, le mage dévoyé; et enfin Robinson Crusoé ${ }^{1}$.

Le trickster et Gribouille

Le trickster, «fripon divin», joueur de tours pendables, d'une activité aussi désordonnée qu'incessante, d'une sexualité débordante, etc., est un personnage probablement universel. Paul Radin (1956) y voyait un miroir de l'esprit, speculum mentis. Il s'agit d'un être fruste et rusé, plein d'innocence et de convoitise, qui enfreint toutes les règles, commet toutes les maladresses, déclenche toutes les catastrophes et tombe dans tous les pièges, y compris ceux qu'il a tendus lui-même. Le parcours du trickster est celui d'un apprentissage par l'absurde, en quelque sorte. C'est au terme de cet apprentissage qu'il deviendra un être humain, ce qu'il n'était pas, ou pas toujours, au départ. Il peut passer une partie de son existence sous forme animale, ou encore le personnage peut être décomposé en plusieurs rôles dont certains sont tenus par des animaux comme la Corneille, le Renard, en Amérique le Coyote, etc. Le trickster est à vrai dire tellement divers, tellement polymorphe qu'il est parfois difficile de lui conserver une réelle individualité. Celle-ci tient peut-être seulement à ce qualificatif de trickster (littéralement "farceur ») qui lui est donné dans les contes des Indiens d'Amérique du Nord où les ethnologues l'ont découvert. Dans d'autres régions, il n'y a pas nécessairement un trickster mais toute une série de personnages qui s'en partagent les caractéristiques. Tels sont par exemple certains dieux et héros de la Grèce ancienne, Athéna, Héphaïstos, Hermès, Prométhée..., dans lesquels la 
recherche comparative a immédiatement reconnu des variantes du trickster dès que celui-ci fut identifié. Ces dieux et ces héros ont en commun l'usage de la raison rusée, la mètis.

6 En Occident, le trickster n'a pas terminé sa carrière avec l'Antiquité, tant s'en faut. On en a reconnu les traits dans d'innombrables personnages de conte, de romans, de comédies. Dans son ouvrage classique sur le sujet, The Trickster (1956), Paul Radin évoque lui-même les clowns de l'Angleterre médiévale -ce sont nos fous, qu'on appellera bouffons à partir du XVIe siècle, du nom d'un personnage de l'opéra italien. Dans le même ouvrage, Karl Kerényi, dont la contribution porte sur la mythologie grecque, mentionne les farces obscènes, phlyakes, qu'on jouait dans les villes doriennes d'Italie du sud, le roman picaresque espagnol, Nasreddin Hodja, Karaghiozis, l'idiot malicieux de la Grèce moderne, et des personnages contemporains comme Félix Krull de Thomas Mann. À cette liste déjà longue, le psychanalyste C. G. Jung ajoute encore Tom Pouce, Jean-le-Sot, les Poltergeister allemands, Polichinelle et quelques-uns de ses comparses de la comédie italienne... Sans oublier les personnages de la bande dessinée, dont un article de 1953, publié fort curieusement dans The Lancet, signale déjà l'intérêt à cet égard.

7 Il me semble que Gribouille, dont la première mention littéraire se trouverait dans un Sermon des fous de 1548, appartient de plein droit à la lignée des tricksters, même s'il n'est qu'un trickster partiel en quelque sorte. Notre Gribouille national se jette à l'eau pour éviter la pluie, mais ce genre d'anecdote est absolument international. Un gribouille chinois, dont j'ai oublié le nom et la référence, marque soigneusement sur le bord de la barque l'endroit exact où son épée est tombée à l'eau, croyant ainsi pouvoir retrouver l'objet plus tard. Un gribouille troupier, le sapeur Camember (1896), reçoit l'ordre de creuser un trou pour y enterrer des ordures. Il s'exécute, puis s'interroge : que faire de la terre du trou? Consulté, l'adjudant lui donne l'ordre de creuser un autre trou... et finit par lui coller quatre jours " pour n'avoir pas creusé le deuxième trou assez grand pour pouvoir y mettre sa terre avec celle du premier trou ». J'ai longtemps cru cette anecdote inventée par Georges Colomb (dit Christophe), l'auteur des Facéties $d u$ sapeur Camember, jusqu'à ce que je la retrouve en feuilletant je ne sais plus quel recueil de contes arabes ou persans. Et que dire de l'étonnement de Bécassine, gribouille au féminin, qui s'étonne qu'il n'y ait pas plus de garçons que de filles à la noce, alors que chaque fille est assise entre deux garçons?

8 Ces petites démonstrations par l'absurde sont évidemment de tous les pays et de tous les temps, encore que... Mais nous manquons du corpus qui nous permettrait d'en évaluer l'importance et d'en analyser le contenu. Leur héros, quand c'en est un, allie les qualités contradictoires qui sont celles du trickster: ruse et niaiserie, malice et innocence. Grand dadais ou petit malin, Gribouille est d'abord un(e) ingénu(e) dont les efforts inventifs sombrent régulièrement dans le ridicule, mais qui réussit parfois là où on ne l'attendait pas, à force d'innocence pour ainsi dire. Une innocence qui révèle alors tout ce qu'il y a d'incohérence et d'arbitraire derrière les conventions qui nous paraissent les plus raisonnables mais qui ne sont en réalité que familières.

On ne peut terminer avec ce personnage sans évoquer les nombreux gribouilles de la bande dessinée. Le plus célèbre en France depuis une trentaine d'années est certainement Gaston Lagaffe, qu'il est inutile de présenter au lecteur. À l'instar de Camember, de Bécassine et de ses autres prédécesseurs, Gaston Lagaffe est un trickster à l'usage des enfants, ce qui n'empêche pas de nombreux adultes de s'en délecter. Il lui 
manque certaines dimensions du personnage, en particulier la sexualité et l'obscénité. Mais il lui en reste assez pour qu'on puisse l'identifier sans le moindre doute. Gaston Lagaffe est bien membre de cette longue lignée des gribouilles qui représentent divers aspects du trickster dans les sociétés européennes.

Le savant fou

Mais le personnage le plus caractéristique, le véritable trickster des temps modernes, c'est sans doute le savant fou -qui d'ailleurs n'est pas fou mais irresponsable ou asocial. Le prototype en est le très célèbre docteur Frankenstein de Mary Shelley (1818). Il n'est peut-être pas tout à fait le premier de sa lignée, encore qu'il n'ait pas de précédent connu au XVIIIe ni au XVIIe siècle. Mais il est à coup sûr le premier et peutêtre le seul à avoir atteint une célébrité aussi durable et étendue, même si on le confond souvent avec sa créature. Le Dr Frankenstein a eu de nombreux successeurs comme le Dr Moreau de H. G. Wells (1896), le professeur Persikov de M. Boulgakov (Les CEufs fatidiques, 1925) ou le Dr Lerne de Maurice Renard (1908). Le roman récent de M. Crichton, Jurassic Park (1990), montre que la veine est loin d'être épuisée. Il s'agit d'une histoire de savant fou tellement classique qu'on la croirait directement reprise de l'île du Docteur Moreau ou des Cufs fatidiques par une simple transposition dans l'actualité de la fin du XXe siècle. La seule particularité est que le personnage du savant fou y est dédoublé : le véritable savant, le généticien qui «fabrique » les dinosaures, n'a qu'un rôle de second plan; le premier rôle revient à l'initiateur de l'affaire, le financier, auquel sont prêtés les traits habituels du personnage.

11 Bon nombre d'histoires de savants fous ont été reprises par le cinéma, qui leur a assuré un retentissement sans commune mesure avec ce qu'aurait été un succès purement littéraire. Ce fut le cas par exemple pour la série des aventures du Dr Fu Manchu, de Sax Rohmer, d'une telle médiocrité qu'on se demande si elles auraient atteint la moindre notoriété sans le cinéma; leur seule originalité était de proposer un savant fou chinois, combinant la peur du péril jaune à celle du progrès scientifique. Mais le cinéma ne s'est pas contenté de diffuser des œuvres littéraires, bonnes ou mauvaises. Il a aussi, très tôt, créé ses propres savants fous que les quelques titres suivants suffiront à rappeler : $L a$ Folie du docteur Tube, d'Abel Gance (1910, ne fut jamais distribué); Le Cabinet du docteur Caligari, de Robert Wiene (1920) et Docteur Mabuse, de Fritz Lang (1922); The Mad Genius, de Michael Curtiz (1931) et Doctor Cyclops, d'Ernest B. Schoedsack (1940); le Dr. Gennessier des Yeux sans visage de Georges Franju (1959); L'Horrible docteur Orloff de Jesús Franco (1961); L'Effroyable secret du docteur Hitchcock de Riccardo Freda (1962), et tant d'autres...

Le cinéma a donc contribué de toute sa puissance d'industrie du spectacle à diffuser le personnage. Peut-être en a-t-il un peu brouillé les traits, dans la mesure où il tend par nature à un certain mélange des genres : les meilleurs films de savants fous sont aussi des policiers, des films noirs ou poétiques, des films d'aventure ou de critique sociale, etc. À l'inverse, un autre mode d'expression dont nous avons déjà parlé, la bande dessinée, tend plutôt à la simplification, voire à la schématisation des récits, et donne souvent au profil du savant fou une particulière netteté. Les savants fous y sont, toutes proportions gardées, sans doute aussi nombreux qu'au cinéma. Je ne me risquerai pas à proposer des titres, même pour mémoire, faute de disposer de sources comparables à celles qui existent pour le cinéma. Je ne ferai qu'une exception pour le comte de Champignac et son ennemi intime Zorglub, avec lesquels le dessinateur Franquin a créé une des paires de savants fous les plus réjouissants de la littérature enfantine, et peut- 
être de la littérature tout court. Des deux, c'est bien sûr Zorglub qui est le véritable savant fou, le comte ne jouant ce rôle qu'épisodiquement.

Cela dit, l'histoire de savant fou la plus épurée que la bande dessinée ait jamais produite est probablement La Marque jaune, d'Edgar P. Jacobs (1956). Et c'est un autre album, Le Manitoba ne répond plus, d'Hergé (1952), qui nous donne, en un raccourci magistral de deux pages, ce qui est à mon sens le schéma élémentaire suivant lequel toutes les histoires de savants fous sont construites. Le schéma qui s'en dégage est le suivant. Le savant fou (qui, rappelons-le, n'est absolument pas fou au sens clinique du terme) :

1) a vu ses idées méconnues, ses projets ridiculisés;

2) il veut prendre sa revanche sur ses confrères, voire sur la société tout entière -ce qui dans les cas extrêmes signifie qu'il veut devenir le « maître du monde »;

3) il doit pour cela travailler à ses recherches en secret, dans un laboratoire clandestin, une usine souterraine, une île...;

17 4) il a néanmoins besoin d'un minimum de collaborateurs, qu'il choisit souvent parmi les asociaux comme lui-même, voire parmi les malfaiteurs;

18 5) mais bientôt, il se met à en fabriquer d'autres: robots, hommes ou animaux artificiels, hommes réduits à l'état d'automates dociles par un quelconque procédé physique ou chimique...

6) une erreur, un incident font soupçonner l'entreprise à l'extérieur ou y font pénétrer un intrus qui essaie de comprendre ce qui s'y passe;

7) mais c'est la trahison ou la révolte de certains des associés du savant, hommes ou robots, qui déclenche la catastrophe,

21 8) dans laquelle le savant fou sera lui-même victime d'une de ses créatures ou d'une de ses inventions.

22 En toute rigueur, je ne peux pas garantir que ce schéma soit le seul possible, ni qu'il soit absolument complet. Il comporte au moins un point faible, le point (6), qui n'est souvent qu'un moyen narratif : l'intervention de l'extérieur n'est pas une nécessité inhérente à la structure même du récit. Peut-être même pourrait-on aller plus loin dans le schématisme, et réduire les histoires de savants fous à trois éléments seulement, qui seraient les suivants :

23 a) le savant croit que ses inventions lui permettent d'outrepasser les lois ordinaires de la nature et de la société;

24 b) il réussit à fabriquer au moins un humanoïde : robot, automate, monstre, zombi, statue animée...;

25 c) à la fin, l'ordre normal reprend ses droits, par une opération de justice immanente : le savant s'est piégé lui-même.

De toute façon, qu'on préfère le schéma en huit points ou le schéma en trois points, il faut les considérer, non comme le résultat définitif de l'analyse d'un corpus, mais comme une grille de lecture provisoire. Et je puis garantir par ma propre expérience que cette grille fonctionne assez bien pour distinguer, dans la masse inépuisable de la littérature fantastique, ce qui est histoire de savants fous de ce qui ne l'est pas. On ne trouvera par exemple qu'assez peu de "vrais " savants fous dans un recueil paru sous ce titre, Les Savants fous, par G. Ponnau (1994), et dans un album plus ancien portant presque le même titre, Le Savant fou, le dessinateur J. Tardi (1977) procède à un mélange des genres avec le policier, le fantastique, etc., où la figure du savant fou, 
d'ailleurs traitée sur le mode parodique, finit par se brouiller. Le Mystérieux docteur Cornélius, de G. Le Rouge (1912-1913) est plutôt un criminel scientifique qu'un savant fou et sert surtout de fil conducteur permettant de tenir ensemble les rebondissements successifs d'un feuilleton à épisodes. Par contre, le professeur Zoran imaginé par Leslie Charteris dans L'Enfer attend le Saint (1959), qui règne sur une armée de zombis dans une ville souterraine cachée à la frontière entre Haïti et Saint-Domingue, est conforme de bout en bout au schéma classique. Et comme j'ai déjà eu l'occasion de le dire, il en est de même pour Jurassic Park. Tout concorde : le laboratoire secret dans une île tropicale perdue au milieu de brouillards continuels; des créatures artificielles, les dinosaures, dont les plus intéressants et ceux qui s'avèreront les plus dangereux, les Velociraptors, ont avec les humains des ressemblances soulignées à plusieurs reprises; un équipement de contrôle informatique ultra-perfectionné mais cependant faillible; et enfin la trahison de l'informaticien, qui déclenche la catastrophe finale en déconnectant les sécurités pour pouvoir voler les embryons congelés qu'il a promis de livrer à une firme concurrente. Tout cela, encore une fois, est rigoureusement conforme au schéma de base.

On vérifie facilement, toujours en appliquant la même grille, que L'Étrange cas $d u$ docteur Jekyll et de Mr Hyde, de R. L. Stevenson (1886), est bien une histoire de savant fou; l'idée originale est que Jekyll se prend lui-même comme sujet d'expérience et que la créature qu'il fabrique est son propre double. Cette idée donne au roman une résonance tout à fait particulière, et même unique, mais le schéma est respecté : le savant finira détruit par sa propre créature. À l'inverse, on peut vérifier tout aussi facilement que, contrairement à une erreur courante que j'ai longtemps commise, le docteur Faust n'est pas un savant fou. Ni l'intervention du diable, ni le jeu entre séduction, perdition et rédemption qui fait l'essentiel du récit, ni surtout l'absence de créatures artificielles (automates, statues animées), de machines, d'inventions, de laboratoire, etc., ne cadrent avec notre grille de référence. Faust n'est pas un savant au sens où le sont nos savants fous. On le décrit comme un homme d'étude, mais on ne nous dit rien sur ce qu'il étudie, si ce n'est que cette «science » ne débouche sur rien de concret. Rien d'étonnant que Faust, dégoûté par une science aussi vaine, doive s'adresser au diable, alors que le savant fou, lui, doit faire face à la puissance bien réelle de ses inventions. Les deux personnages sont radicalement étrangers l'un à l'autre.

Ayant écarté Faust, nous ne trouvons plus dans la littérature européenne de savant fou antérieur à Frankenstein -constat tout provisoire, bien sûr, car il n'est pas dit qu'il n'y ait rien dans la masse gigantesque des écrits oubliés des siècles précédents. Par contre, notre grille nous permet de faire un saut de deux mille ans, et d'identifier un véritable savant fou dans le personnage de Dédale, qui était le patron des artisans en Grèce ancienne. On prêtait à Dédale l'invention de la statuaire anthropomorphe, celle de divers outils, et plusieurs innovations dans le domaine de la métallurgie. Mais il est aussi à l'origine d'un monstre, le Minotaure. Il fabrique des statues animées qu'on peut considérer comme des préfigurations de nos modernes robots, et le Labyrinthe fait penser à nos laboratoires cachés. Sa renommée le conduit au service de plusieurs rois, avec lesquels il a tendance à se fâcher; il fait alors appel à toute son inventivité, soit pour s'enfuir, soit pour se venger. Son fils Icare périt, victime de l'une de ses inventions, et lui-même finira de même. La seule difficulté pour identifier Dédale comme savant fou tient à notre documentation. Nous n'avons pas de récit unique dans lequel Dédale apparaîtrait avec tous les traits caractéristiques du personnage. Nous 
avons à son sujet des sources diverses, fragmentaires, de dates et d'origines différentes, et c'est par un montage peut-être arbitraire de ces sources que nous obtenons un portrait complet. Aussi faut-il faire une importante réserve. Si, et seulement si Dédale a bien été, pour les Grecs anciens, le personnage que nous pouvons reconstituer aujourd'hui, alors il s'agit bien d'un savant fou avant la lettre. La question est de savoir si ce sont bien les Anciens eux-mêmes qui ont composé le personnage, et pas nous. Voilà pourquoi l'authenticité du personnage de Dédale doit être minutieusement vérifiée dans tous ses détails.

Mais si, provisoirement, nous admettons cette authenticité, une nouvelle question se pose. Entre la disparition de Dédale et l'apparition de Frankenstein, plus de quinze siècles s'écoulent, pendant lesquels il semble n'y avoir plus nulle trace du savant fou. Comment comprendre cette longue, cette interminable éclipse ?

Il est difficile de l'imputer à une quelconque pause de l'innovation technique pendant toute cette période, ou à une orientation différente dans l'évolution des techniques. Mais on soupçonne rapidement que les explications sont à chercher du côté de la religion. Dans les histoires de savants fous, le surnaturel n'intervient pas, et la justice est immanente : voilà deux conceptions bien éloignées de celles du christianisme et des religions monothéistes en général. Il est même assez logique de penser que cette incompatibilité s'étend à toutes les religions de transcendance d'un côté, et à tous les personnages du genre trickster de l'autre. Ainsi s'expliquerait par exemple que pendant le Moyen Âge européen, les tricksters ne subsistent que comme personnages burlesques dans certaines fêtes (la Fête des Fous, la Fête des Ânes...) ou dans les spectacles de rue, et sous une forme probablement appauvrie. Quoi qu'il en soit, le rôle du surnaturel est à examiner de plus près. Nous allons le faire en commençant par un personnage que nous venons d'écarter, mais qui va précisément s'avérer d'un grand intérêt: le docteur Faust.

Faust et la fin de la magie

31 Il est établi depuis longtemps que le thème de l'homme qui vend son âme au diable est d'origine byzantine. Alain Ducellier (1976) précise :

«[Ces histoires] sont très fréquentes dans le monde byzantin; l'une d'elles, la célèbre histoire du diacre d'Adana, Théophile, qui se donne au démon et obtient son pardon grâce à l'intercession de la Vierge, impressionna tellement les Occidentaux à l'époque des Croisades qu'elle envahit notre littérature pieuse et notre théâtre sacré, non sans inspirer nos sculpteurs et nos peintres. Dans le même ordre d'idées, on a pu assigner avec quelque vraisemblance une origine orientale au célèbre mythe de Faust. »

Faust n'est pas un savant fou mais un magicien. La magie est, il est vrai, une " action traditionnelle efficace » qu'il est impossible de distinguer de la technique sur la base de cette seule définition, comme Mauss l'avait vu dès 1902. Cependant, la magie n'est pas une croyance universelle et uniforme, située hors du temps. Elle se développe et elle régresse, elle change de contenu, bref elle a une histoire. Et Byzance a écrit un chapitre important de cette histoire. D'après A. Ducellier, le christianisme byzantin est dominé par l'idée que ce monde-ci est soumis à l'intervention réelle des forces de l'au-delà, bonnes et mauvaises. Reliques et icônes captent ces forces et leur font exercer une sorte de protection contagieuse. Rites et sacrements sont des charmes qui permettent de contraindre les forces du bien et jusqu'à Dieu lui-même. L'ordalie, le tirage au sort, l'incubation (dormir avec un objet sacré), l'évocation, le serment ont aussi une efficacité matérielle incontestée. «Magie et sainteté produisent souvent les mêmes 
effets », ce qui rend bien difficile pour le profane de faire la différence. D'autant que les démons sont toujours là, présents partout et sous les déguisements les plus trompeurs, presque aussi puissants que Dieu et ses anges. Dans ces conditions, distinguer les "vrais » miracles des prestiges diaboliques est une affaire délicate, qui nécessite le recours à d'autres rites et à d'autres charmes...

On imagine facilement les abîmes de complexité et de perplexité qui attendent le mortel assez ambitieux pour essayer de voir clair dans tout cela. À Byzance, la science des rites et des miracles est un des plus gros chapitres de la théologie, auquel fait pendant une démonologie non moins élaborée. Sans doute était-ce là la "science » de Faust. Car en Occident aussi, la magie a eu son histoire. Il s'y trouve dès le XIIIe siècle des clercs comme Roger Bacon pour affirmer haut et fort qu'il s'agit d'une croyance vaine, aux effets nuls, parce que rien ne peut se faire dans la nature qui ne soit l'effet de causes naturelles. Mais Roger Bacon lui-même passera plus tard pour magicien, comme son contemporain Albert le Grand, pourtant aussi peu magicien que lui. Contrairement à ce qu'on a tendance à croire spontanément, ce n'est pas au Moyen Âge que la magie prend son plus grand développement en Occident, mais aux XVe et XVIe siècles, avec d'importants prolongements au XVIIe. Ce qui signifie que pendant plus de deux siècles, magie et science se développent parallèlement, comme deux branches également sérieuses du savoir.

L'apogée de la magie en tant que science se situe probablement au début du XVIe siècle. Il se constitue à cette époque un véritable réseau international de "mages " qui échangent leurs informations, voyagent pour se visiter les uns les autres et écrivent des livres dans lesquels ils publient les résultats de leurs recherches. Ces mages sont des clercs, c'est-à-dire des religieux et des savants, et la « haute » magie dont ils s'occupent n'a rien à voir, selon eux, avec la magie populaire qui n'est que du charlatanisme. Après de longues années d'efforts, tous ou presque devront reconnaître leur échec.

La plupart rentrèrent dans le rang et redevinrent de simples fils obéissants de l'Église avec laquelle ils n'avaient jamais voulu, ni cru rompre. D'autres peut-être tombèrent dans le charlatanisme. On comprend que dans ce contexte, le vieux thème byzantin du pacte avec le diable ait éveillé de nouvelles et fortes résonances. Dans le réseau de mages que je viens d'évoquer, apparaît vers 1500-1510 le nom d'un certain Georgius ou Johannes Faustus, dont on ne sait pas grand-chose sinon qu'il s'occupait de nécromancie. Quatre-vingts ans plus tard, en 1587, paraît à Francfort-sur-leMain la première version littéraire connue de l'histoire du docteur Faust. Presque immédiatement (il meurt en 1593), le dramaturge anglais Christopher Marlowe en tire une pièce qui le rend célèbre, The Tragical History of Doctor Faustus. Retraduite en allemand, elle connaîtra un succès exceptionnel en Europe centrale et, par exemple, restera au répertoire du théâtre populaire de Vienne jusqu'au XVIIIe siècle. En Espagne, Pedro Calderón de la Barca reprend le thème dans sa pièce, El Mágico prodigioso, en 1637. Mais il le traite dans une perspective différente, directement inspirée par la théologie du concile de Trente. Celle-ci, en effet, met une limite infranchissable à l'action des forces diaboliques en ce monde : le libre-arbitre. Les pouvoirs de l'enfer sont réduits à ceux de la séduction et de l'illusion -c'est presque la même chose- et avec la grâce de Dieu, chacun a les moyens de s'en défendre pourvu qu'il le veuille.

La magie, en somme, est attaquée de deux côtés. Prise au sérieux, elle est soumise par les savants à un examen auquel elle ne résiste pas. Et dans le même temps, les théologiens, qui sont eux aussi des savants, la réduisent à une entreprise de séduction 
d'ordre purement psychologique, pour employer un terme un peu anachronique dans ce contexte. Bientôt, on cessera complètement de prendre la magie au sérieux. Les lois qui la réprimaient seront abolies ou tomberont en désuétude. Il subsistera certes des croyances populaires, mais dont il est difficile de dire l'importance faute de points de comparaison historiques. Pour le reste, la magie deviendra l'art du spectacle que nous connaissons aujourd'hui, fait de trucages, de prestidigitation et de physique amusante, où le surnaturel, lorsqu'il est encore évoqué, ne l'est plus que comme procédé de mise en scène. Cet art a d'ailleurs un intérêt considérable pour la Technologie (Sigaut 2002), mais ce n'est pas le lieu de développer ce point de vue ici.

L'histoire de Faust, en définitive, c'est la fin de la magie en Occident. Dans les sociétés où on ne fait pas de distinction fondamentale entre nature et surnature, on ne peut pas non plus distinguer la technique de la magie (c'est ce qu'avait vu Mauss), et l'histoire de Faust n'a pas lieu d'être. Elle n'en a pas non plus dans des sociétés comme la nôtre où la croyance au surnaturel, lorsqu'elle existe, est un choix personnel qui n'affecte nos actions que dans le domaine de la psychologie, pour employer encore une fois le terme d'une façon un peu impropre. L'histoire de Faust n'a de sens que dans des sociétés où l'on croit en une surnature, mais où nos rapports avec elle ne vont plus de soi. Où, en somme, il n'est plus tout à fait naturel d'avoir affaire au surnaturel. Où donc, enfin, technique et magie vont être distinguées, non pas dans la pratique (elles l'étaient déjà), mais dans la pensée spéculative. Voilà pourquoi le personnage de Faust nous intéresse ici. Il n'a rien à voir avec celui du savant fou, mais si l'absence de ce dernier sur la scène européenne pendant plus de quinze siècles est avérée, il nous aide à comprendre que cette absence a sans doute des implications plus profondes qu'il n'y paraît à première vue.

Transcendance, travail et création

Nous avons vu que dans les histoires de savants fous et de tricksters, l'invention n'est pas bonne ou mauvaise, elle est bonne et mauvaise, indissociablement. Elle est bonne parce qu'elle satisfait des désirs humains, en quoi aussi elle est inéluctable : l'homme peut aussi peu se passer d'inventer que de respirer. Mais elle peut devenir mauvaise, comme le désir lui-même quand il ne se connaît plus de bornes. Pour devenir une technique, l'invention doit être socialisée, ou pour mieux dire domestiquée : c'est précisément ce à quoi le savant fou se refuse, par orgueil ou par ambition, et c'est en cela que consiste sa folie. En toute rigueur, il faudrait appeler le savant fou un inventeur asocial, mais je me garderai bien de proposer une expression aussi manifestement vouée à l'insuccès! Or, comme nous l'avons vu à propos des rapports au surnaturel, cette conception de l'invention aussi dangereuse qu'inévitable, procédant du désir et cause de désordre, s'accorde mal avec les valeurs enseignées par les grandes religions. Celles du bouddhisme par exemple, qui professe l'extinction des désirs. Celles du judaïsme et de l'islam, qui professent la soumission à des traditions indépassables. Celles du christianisme enfin, pour tout un ensemble de raisons dont les unes sont semblables et les autres différentes. Le christianisme enseigne aussi le contrôle des désirs et la soumission à l'autorité. Mais il enseigne également une valeur qui, sans lui être absolument particulière, est assez rare en dehors de lui : la sainteté du travail. Il existe un plan de Dieu sur le monde. Par son travail, qui est aussi son devoir d'état, l'ouvrier collabore à ce plan. Et il est assez facile d'imaginer comment cette idée a pu se laïciser pour devenir celle du progrès. Dans cette vision des choses, l'invention devient le fruit du travail industrieux. Elle est, en quelque sorte, domestiquée d'avance, et ses méfaits deviennent de simples inconvénients 
qu'un progrès futur ne tardera pas à corriger. Le génie est un don de la Providence, le savant est d'abord un sage.

D'après un intéressant essai de John T. Mason (1988), toutes ces idées se rattachent à une conception unitaire de la création, qui fait de celle-ci l'acte pur et immatériel d'une volonté toute puissante. Cette conception apparaît dans la Bible, avec le premier récit de la création du monde dans la Genèse, récit appartenant à la tradition dite «sacerdotale » et qui aurait été composé vers la fin du VIe siècle avant notre ère. Elle apparaît un peu plus tard mais de façon apparemment indépendante dans deux philosophies grecques : le néo-platonisme et le stoïcisme. Saint Paul et les premiers Pères de l'Église, en particulier saint Augustin, réuniront ces deux traditions en une synthèse qui sera intégrée au dogme chrétien. Le Tout-Puissant est nécessairement l'Unique, sinon sa toute puissance serait bornée. Et pour la même raison, il a aussi le monopole de la vérité et de la justice. Il tire le monde et les êtres du néant par l'exercice de sa volonté pure : il n'a pas besoin de matière préexistante à façonner, ni d'aucun instrument. Le processus même de la création est strictement immatériel. Il n'y a ni chaos primitif, ni succession de mondes tout à tour détruits et reconstruits, ni une population de dieux avec leurs activités, leurs rapports entre eux et avec les humains (y compris sexuels et conflictuels), ni conséquences fâcheuses expliquant le mal, le malheur, la mort... Rien qu'une parole et la contemplation satisfaite de son résultat : «Dieu dit : "Que la lumière soit", et la lumière fut. Dieu vit que la lumière était bonne, et Dieu sépara la lumière et les ténèbres. Dieu appela la lumière "jour" et les ténèbres "nuit". Il y eut un soir et il y eut un matin : premier jour ».

Cette conception, pour grandiose qu'elle soit, a l'inconvénient de rendre l'existence du mal absolument inexplicable, à moins d'avoir recours à des contorsions rhétoriques qui la défigurent. Sans doute est-ce pour cette raison qu'elle n'a pas pu prévaloir entièrement dans la Bible même, où le premier récit de la création est suivi d'un autre, plus ancien de deux à trois siècles, qui incorpore de nombreux éléments de mythologies plus primitives. C'est dans ce second récit, appartenant au courant dit yahviste, que Dieu se sert d'argile pour façonner l'homme et de son propre souffle pour l'animer. Ensuite interviendront le serpent, le péché, la chute... Mais le problème du mal restera intraitable et inspirera entre autres le Livre de Job (probablement du IVe siècle), dans lequel un personnage sans doute d'origine iranienne, Satan, fait sa première apparition biblique.

Mais tout cela est une autre histoire. Ce qui nous importe ici est de distinguer deux grandes traditions concernant la création, qui est en quelque sorte l'image divine de l'invention. J.T. Mason résume dans le tableau suivant les caractéristiques que prêtent ces deux traditions à l'acte de création :

\begin{tabular}{|l|l|}
\hline Tradition dualiste & Tradition moniste \\
\hline \hline Pluralité & Unité \\
Changement & Stabilité \\
Désordre & Ordre \\
Conflit & Harmonie \\
Matérialité/sexualité & Spiritualité \\
\hline
\end{tabular}


Pour ma part, je serais enclin à ajouter aux deux colonnes du tableau les termes suivants

\begin{tabular}{|l|l|}
\hline Imprévisibilité du futur & Progrès \\
\hline \hline Immanence & Transcendance \\
\hline
\end{tabular}

«Immanence », on l'aura compris, signifie ici qu'il n'y a pas de surnature ou que, s'il y en a une, elle a ses propres nécessités et ses propres limites. Il peut y avoir des puissances supérieures, occultes ou non, il n'y a pas de toute puissance absolue. La surnature n'est en somme qu'une autre nature ou un autre domaine de la nature. «Transcendance» exprime l'idée inverse: la nature que nous connaissons est totalement soumise à une entité supérieure et la nécessité physique elle-même n'est qu'une des manifestations de cette soumission.

Mais nous devons prendre garde que nos deux traditions sont des reconstructions a posteriori. On a certes utilisé des matériaux historiques pour les élaborer, mais on ne peut pas pour autant les considérer comme des réalités historiques, présentes telles quelles à tel moment dans tels esprits. Il faut plutôt les considérer comme des grilles de lecture, permettant de ranger dans un ordre sans doute provisoire des matériaux toujours partiels et disparates. La tradition dualiste, la plus ancienne, est aussi la plus universelle. On la trouve un peu partout, mais sous des formes si différentes que ce sont ces différences qui importent plutôt qu'une présence qui, à elle seule, n'est guère significative. La tradition moniste au contraire est plus rare et lorsqu'elle est présente, ne l'emporte jamais complètement dans tous les domaines. C'est plutôt une tendance, un rêve, voire un cauchemar, qui lorsqu'on tente de l'appliquer jusqu'au bout dans une société réelle ne peut aboutir qu'à des conflits particulièrement implacables. Par la force des choses, en somme, on a l'impression que le monisme ne peut exister que comme projet, ce qui n'est pas vraiment exister, ou qu'à l'intérieur de certaines limites, et ce n'est plus tout à fait un monisme. Ironique revanche de l'immanence sur la transcendance...

Robinson Crusoé

Nous n'en avons pas encore fini avec les personnages de fiction. Il nous en reste un, particulièrement important, Robinson Crusoé. Avec lui, nous ne quittons pas les parages de la philosophie. Les lecteurs de Techniques \& culture (9, pp. 167-174) se souviennent peut-être que le premier à faire usage du paradigme de l'île déserte fut, pour autant qu'on le sache, Leibniz. Il s'agissait pour lui de se représenter « de combien de lumieres on auroit besoin pour se pouvoir fayre à soy même [...] tout ce qui peut nous fournir d'utile et de commode l'abondance d'une grande ville toute pleine des meilleurs ouvriers... ». Une expérience mentale, en somme, pour laquelle on imagine que tout l'héritage humain a disparu et qu'il faut le reconstituer pas à pas, pièce à pièce... Prise au pied de la lettre, l'expérience est évidemment irréalisable. Si Robinson était vraiment un nouvel Adam, il lui faudrait autant de temps qu'à la lignée entière de ses ancêtres pour retrouver tout son héritage. Mais considérée comme un jeu, l'expérience est fascinante. Elle a longtemps passionné les enfants (je ne sais trop si c'est encore le cas) et quoi qu'ils en aient, elle passionne aussi la plupart des chercheurs 
en sciences humaines. Car que cherchent-ils d'autre qu'à rendre explicite telle ou telle parcelle de cet héritage dont nous vivons tous sans y penser?

Nous avons heureusement, sur Robinson Crusoé, une belle étude de B. Rupp-Eisenreich (1987) qui montre sa place tout à fait centrale dans la pensée européenne du XIXe siècle, notamment en Allemagne. Cette étude me permet, en y renvoyant le lecteur, de me borner aux quelques remarques ici essentielles pour mon propos.

Commençons par préciser les contours du personnage, en particulier par rapport au savant fou. Robinson et le savant fou sont deux techniciens, en ce sens que leur savoir est efficace. Ce sont aussi deux solitaires, quoique leurs solitudes soient bien différentes : celle du savant fou est voulue, c'est un moyen de se cacher d'une société dans laquelle il entend revenir en triomphateur; celle de Robinson est subie et il fait tous les efforts possibles pour en sortir. À partir de là, tout les oppose. Le savant fou est un inventeur qui prend pour lui-même et les autres des risques insensés en manipulant des forces inconnues sans précautions suffisantes; Robinson est un travailleur qui aménage son île déserte, c'est-à-dire qui la soumet à l'ordre d'une société dont il se trouve être l'unique représentant. Le savant fou ne croit pas en Dieu ou ses recherches le lui font oublier; Robinson revient à Dieu dont son esprit aventureux l'avait éloigné (il y a en lui du fils prodigue). L'histoire du savant fou est celle d'une chute, celle de Robinson d'une rédemption. Sur son île, Robinson va retrouver toutes les lumières dont parle Leibniz, plus les valeurs indissociables de la prière et du travail; il réinvente en somme la règle de saint Benoît, ora et labora. En un mot, Robinson est le héros positif par excellence, où l'on reconnaît sans peine plusieurs des valeurs chrétiennes dont il a été question plus haut, en particulier la sainteté du travail.

Il est rare cependant que les héros positifs passionnent le public, et si Robinson n'avait été que cela, il n'aurait sans doute pas fait une aussi belle carrière. Il y a d'ailleurs de nombreux Robinsons qui n'ont été que cela, et dont les plates aventures sont d'un ennui profond. Il y a donc autre chose, qu'il faut essayer de déceler.

Revenons brièvement sur la chronologie. Robinson Crusoé paraît en 1719, 130 ans environ après Faust (1587) et presque exactement un siècle avant Frankenstein (1818). Mais on a vu que Faust et Frankenstein avaient des précédents plus ou moins lointains. Robinson, lui, ne semble pas en avoir. Il y a depuis fort longtemps - au moins depuis l'Odyssée- une littérature maritime où les histoires de naufrages abondent; ce sont en général des prétextes à merveilleux sans rapport avec notre sujet. Plus importantes sont les histoires d'enfants sauvages ou grandis dans un isolement humain complet (enfant-loups, Kaspar Hauser), qu'on trouve également dès l'Antiquité. Les unes, qui traitent de cas réels ou qui cherchent à établir leur réalité, ne relèvent pas de la fiction et n'ont donc pas leur place ici. Les autres, qui traitent de cas imaginaires, sont des apologues philosophiques. Mais dans presque tous les cas, la question posée est celle de la part innée ou acquise, individuelle ou sociale, de la nature humaine. Dans un cas, même, l'histoire n'est qu'un prétexte à démonstration : il s'agit de Hayy bin Yaqzân, le "philosophe autodidacte " de l'auteur andalou Ibn Tufayl (XIIe siècle). Abandonné sur une île déserte à sa naissance (ou né par génération spontanée, ce qui est encore mieux), d'abord allaité par une gazelle, le héros va progressivement, en sept chapitres de sept ans chacun qui le conduisent au seuil de la cinquantaine, redécouvrir à lui seul et par le simple exercice de sa raison, toute la philosophie de l'auteur de A à Z. Il n'y a là, de toute évidence, qu'un procédé pédagogique pour donner une apparence de naturel à ce qui est en réalité un cours de philosophie. Ces 
remarques n'ôtent rien à l'intérêt du livre pour une histoire de la philosophie dans le monde arabe au Moyen Âge. Mais nous ne sommes plus dans notre sujet, tant il est clair que l'idée de se demander ce qui aurait bien pu arriver à un enfant réel dans une île réelle n'a pas un instant effleuré l'auteur.

Or c'est sans doute là que gît le lièvre. Robinson n'est pas un enfant et encore moins un nourrisson. C'est un homme adulte et un marin parce qu'il fallait qu'il le fût pour que son histoire paraisse véridique. Et il fallait qu'elle paraisse véridique pour permettre à des générations de lecteurs de se l'approprier, d'en faire le thème de leurs jeux et de leurs rêves. Il est tout à fait improbable que Daniel Defoe ait lu Leibniz, dont bon nombre d'écrits n'ont été publiés que longtemps après sa mort. En revanche, Defoe est reconnu comme le père du roman réaliste anglais. Il ne se mit à la fiction que sur le tard, après une longue carrière de pamphlétaire et de journaliste. Pour lui, la meilleure fiction était celle qu'on ne pouvait pas distinguer du récit authentique d'un témoin oculaire. Et dans son œuvre, il réussit si bien à mélanger les deux que jusqu'à nos jours, les experts s'y sont parfois trompés...

Robinson Crusoé est un personnage réaliste. Et il ne l'est pas par un simple artifice de narration destiné à le rendre plus crédible. Il est réaliste par nécessité, par raison d'être. Il n'aurait pas d'intérêt et donc pas d'existence s'il ne l'était pas. Et c'est très probablement parce qu'il est réaliste qu'il est sans précédent. Il est vrai que j'en sais trop peu sur l'histoire du réalisme en littérature pour pouvoir établir cette assertion sur des bases solides. Je me rallie sur ce point aux commentateurs de Defoe, qui font en général de lui l'inventeur du réalisme littéraire.

Pour faire mieux entendre ce que je veux dire avec le réalisme par raison d'être, on me permettra une brève comparaison avec le roman policier. Là également, le réalisme n'est pas seulement un procédé narratif. Il ne s'agit pas seulement de décrire de façon vraisemblable un crime et la recherche du ou des coupables. Il s'agit (en principe) de donner au lecteur les moyens de trouver lui-même la solution. Il faut lui donner l'impression qu'il participe à l'enquête, qu'il la mène, qu'il doit lui-même élucider l'affaire...

53 Dans les histoires de savants fous, au contraire, le réalisme n'est plus qu'un simple procédé de narration. Ce réalisme-là porte sur les circonstances, sur le décor, il cherche à produire une atmosphère d'authenticité. Mais c'est pour mieux conduire à l'incroyable. Car les histoires de savants fous sont incroyables par nature. Elles relèvent de ce que Maurice Renard appelait en 1909 le merveilleux scientifique, et qui est devenu la science-fiction. Dès qu'on en arrive aux inventions du savant, en effet, il n'y a plus de réalisme possible puisque, par définition, ces inventions ne peuvent pas être décrites dans des termes véritablement scientifiques. Et si d'aventure un auteur voulait le faire, il se trouverait vite embarqué dans un pseudo-traité de physique, de chimie ou de biologie qui aurait peu de chances d'attirer plus de lecteurs que les vrais. De tels pseudo-traités existent, du reste, mais ils appartiennent à un domaine tout différent, celui du canular scientifique. Élaborer des hypothèses fausses, mais assez ingénieuses pour surprendre, amuser, intéresser par elles-mêmes est un exercice difficile, qui ne peut guère être goûté en dehors du cercle des spécialistes concernés. Il y a un peu de cela dans Jurassic Park, mais seulement parce que les bugs de l'informatique et le clonage de l'ADN sont des sujets à la mode. En règle générale, les inventions du savant fou restent enfermées dans leur boîte noire, que le réalisme ne peut ni ne doit ouvrir. 
Pour aller au bout de cette discussion, il nous faudrait nous interroger sur la fiction une combinaison de narration et de jeu ?- et surtout sur ses rapports à ce «réel »si difficile à définir. Disons seulement que pour qu'une fiction trouve des auditeurs, des spectateurs ou des lecteurs, ce qui est sa condition d'existence, il faut bien qu'elle leur communique cette intuition, cette sensation de réalité dont a parlé A. Michotte, et qui est parfaitement compatible avec la conscience claire que cette réalité n'existe pas. $\mathrm{Si}$ on donne ce sens très large au terme de "réalisme ", alors toute illusion, toute fiction doit être réaliste pour être réussie. Mais toutes ne sont pas réalistes de la même façon, par les mêmes moyens. C'est ce qui justifie le sens beaucoup plus étroit que nous avons donné au réalisme dans le cas du Robinson Crusoé et du roman policier. Il est probable que les différents arts de la fiction, le conte, le spectacle (théâtre, cirque...), le roman, le cinéma, etc., n'emploient pas les mêmes moyens d'illusion, bien que je ne sois pas capable, pour ma part, de suggérer en quoi consistent leurs différences. Mais ce qu'il est loisible à chacun d'observer, c'est que nos trois héros, le savant fou, Faust et Robinson, ont des profils médiatiques bien différents, si on me passe l'expression. Robinson a fait presque toute sa carrière dans le roman, on l'a peu vu au théâtre ou au cinéma. Faust est un personnage de théâtre, y compris l'opéra, qui n'a fait qu'une carrière assez courte au cinéma et qui semble absent du roman. Quant au savant fou, son succès a commencé dans le roman, mais ne s'est guère étendu au théâtre; ce sont le cinéma et la bande dessinée qui ont porté sa popularité au pinacle. Si nous mettons ensemble toutes ces différences, y compris celles qui concernent la chronologie, nous obtenons le tableau suivant :

\begin{tabular}{|l|l|l|l|}
\hline & Faust & Robinson & Le savant fou \\
\hline \hline Date d'apparition & 1587 & 1719 & 1818 \\
\hline \hline Précédents & magiciens & aucun & Dédale \\
\hline \hline & byzantins & & \\
\hline \hline & théâtre & & roman \\
\hline \hline Profil médiatique & opéra & roman & cinéma \\
\hline \hline Degré de « réalisme » & minime & essentiel & accessoire \\
\hline \hline
\end{tabular}

Nous avons, en somme, différents personnages qui cristallisent certaines préoccupations d'une époque, et différents arts, qui ont chacun leur formule d'illusion. Et pour comprendre vraiment ce qui se passe, nous aurions besoin de savoir en quoi chaque personnage est plus ou moins en adéquation avec la formule propre à chaque art. On imagine facilement, par exemple, que la formule du roman, qui suppose le plus souvent la lecture solitaire et silencieuse, doive différer de celle du théâtre, qui suppose des acteurs, un décor et un public. Le théâtre a d'ailleurs d'innombrables sous- 
catégories, chacune avec leurs conventions différentes, sans parler du théâtre d'ombres, des marionnettes, du cinéma...

On parle souvent de pensée populaire, sans toujours préciser ce qu'il faut entendre par là. Peut-être voit-on mieux maintenant de quoi il s'agit. Nous ne pouvons rien dire d'une pensée qui ne s'exprimerait pas, ou qui ne s'exprimerait que dans les conversations quotidiennes sans être recueillie et mise par écrit. Aujourd'hui, ce recueil est la tâche des psychologues, des anthropologues, voire des journalistes. Avant eux, nous avons eu des mémorialistes, des essayistes, etc. Mais tout cela ne nous permet pas de remonter très loin dans le passé, surtout celui des classes non dominantes. Pour nous, la pensée populaire ne peut être que celle qui s'exprime dans les différents arts de la narration et du spectacle. D'où la nécessité de connaître les conditions de production de ces arts, leur fonctionnement, ce que j'ai appelé leur formule d'illusion. Car ces conditions ont de toute évidence un effet considérable sur leur contenu. C'est sans doute en grande partie pour cette raison que Faust a atteint le succès par le théâtre et Robinson par le roman. Le réalisme de Robinson a d'ailleurs pour référence le récit objectif qui est aussi la forme canonique de l'exposé scientifique. Dans la mesure où ce réalisme est une de ses conditions d'existence, on comprend mieux que Robinson n'ait pas eu de précédent avant l'époque moderne.

L'histoire de Robinson Crusoé a des significations multiples. Certaines d'entre elles ont été reprises récemment par des auteurs contemporains, soit dans le registre de la fiction, soit dans celui de l'essai ou du commentaire. Les significations qui nous concernent ici sont celles qui relèvent de la pensée sur les techniques. Robinson Crusoé, on l'a vu, c'est l'expérience mentale de Leibniz transposée sur le mode romanesque : plaçons-nous sur une île déserte, de quoi avons-nous besoin pour y vivre comme chez nous? Quelle est la somme de savoirs nécessaires à la vie moderne ? Peut-on en faire l'inventaire, « on » étant quelqu'un, c'est-à-dire un individu ?

Je crois que le roman de Defoe donne deux réponses contraires à la dernière de ces questions. La première est : oui, un individu peut reconstruire sa société à lui seul ou peu s'en faut, et c'est l'histoire telle qu'elle nous est racontée. Mais c'est une réponse truquée. Elle n'est possible que parce que l'auteur a mis une complaisance plus que suspecte à fournir à son héros toute la quincaillerie du navire échoué. Supprimons cet artifice, nous dit Jules Verne dans un roman ironique et peu connu, L'École des Robinsons (1882), et il n'y a plus d'histoire possible. Nos deux Robinsons (ils sont deux), bien qu'ils aient tout de même à leur disposition un assez bon couteau, ne peuvent que survivre misérablement en mangeant des œufs d'oiseaux de mer et des coquillages crus. Ils ne sont même pas capables d'allumer du feu avec deux morceaux de bois, comme le moindre sauvage sait le faire. Aussi leurs aventures tourneraient-elles court... si l'auteur ne faisait intervenir d'autres éléments que je me garderai bien de dévoiler.

59 À mon sens, l'artifice de Defoe équivaut à une réponse négative : non, un homme seul ne peut pas reconstruire sa société. Le partage des tâches et des savoirs a atteint un niveau tel que la part de chacun est devenue insuffisante pour le lui permettre. La société n'est plus dans les individus. C'est une réalité d'un autre ordre. Et dans 1a masse de plus en plus immense des savoirs qu'elle met en œuvre, la part de chacun devient de plus en plus négligeable. La technique suppose le contact avec la matière, avec la nature, et dans ce sens Robinson est le technicien par excellence. Mais à l'époque où écrit Defoe, le machinisme a déjà fait des progrès dont s'émerveillent un Leibniz, et un Charles Perrault (1688), entre autres. La société devient de plus en plus 
technicienne, mais les individus qui la composent le sont de moins en moins, à la fois parce que la part de chacun dans le savoir commun tend à se réduire de plus en plus, et parce que le contact avec la matière ou avec la nature, qui est l'essence même de la technique, devient de plus en plus indirect et lointain du fait de l'interposition des machines. C'est ce paradoxe apparent que Defoe révèle en cherchant à le dissimuler, me semble-t-il. P. Pellegrin (1982) a suggéré, à propos de la Politique d'Aristote, qu'« une réalité sociale ne devenait vraiment pensable qu'après son abolition ". J'ai l'impression que c'est exactement ce qui s'est passé avec Robinson Crusoé. Lorsque Defoe publia son roman, il y avait certes longtemps que la société n'était plus à l'échelle des individus. Mais rares étaient ceux qui en avaient conscience, et on ne voit pas comment leurs réflexions auraient $\mathrm{pu}$ atteindre le public. Le machinisme va rendre cette réalité tangible pour tous. Et c'est probablement pour cela que tout le monde va se passionner pour une histoire devenue manifestement impossible, sauf dans la fiction.

Techniques et narration

Il n'y a pas une pensée populaire et une autre qui ne le serait pas. Il n'y a que des modes d'expression qui diffèrent par leur forme et par leur contenu. Ce que, par commodité ou relâchement d'attention, nous appelons parfois pensée populaire, est avant tout une pensée narrative ou poétique au sens propre du terme. Il s'agit de faire rire ou de faire peur, d'amuser ou d'instruire, d'émouvoir et toujours d'intéresser un public d'enfants ou d'adultes, un auditoire rassemblé autour du conteur ou des lecteurs lisant chacun dans le silence de leur chambre. Mais dans tous les cas, il faudra raconter de belles histoires qui fassent rêver en créant l'illusion. La nature de l'illusion -ce qui est croyable et ce qui ne l'est pas- varie d'une société à l'autre, de même que les moyens de l'illusion varient d'un mode narratif à un autre. Mais il faut qu'il y ait illusion, et pour qu'il y ait illusion il faut qu'il y ait un minimum de réalisme (ce réalisme au sens large dont le réalisme littéraire de Defoe est un cas particulier). En d'autres termes, il ne peut pas y avoir de belle histoire sans un minimum de matériaux empruntés au monde quotidien et familier du public auquel on s'adresse. Parmi ces matériaux, les techniques ont leur place. Mais elles ne sont pas marquées comme telles, parce que la pensée narrative, qui n'est ni analytique ni démonstrative, n'a que faire du concept de technique. Nous serions mal venus de lui en faire le reproche. C'est à nous de l'y trouver, si cela nous intéresse et si nous jugeons que cela en vaut la peine.

61 Est-ce que cela en vaut la peine ? La réponse ne va pas de soi, surtout si on prend la mesure de l'immensité du domaine à explorer. Nous n'y avons fait qu'une brève incursion. Par commodité, j'ai choisi de suivre quelques personnages qui, à tort ou à raison, m'ont paru plus techniciens que d'autres : le trickster, le savant fou et Robinson Crusoé. Mais aucun d'eux n'est seulement technicien -heureusement !- et ils ne sont pas les seuls à l'être. Je pense par exemple au personnage de Pygmalion, dont l'importance est certainement du même ordre. À l'origine, l'histoire est celle d'un sculpteur qui tombe amoureux de la statue qu'il a faite, Galatée, au point qu'à force de prières, il obtienne d'Aphrodite qu'elle lui donne vie. Mais en réalité, cette histoire est celle de tous les constructeurs amoureux de leur œuvre au point d'oublier pour quoi ou pour qui elle est faite. Et si nous la prenons ainsi, alors la littérature moderne a produit de nombreux Pygmalions. Deux d'entre eux, particulièrement attachants, sont le colonel Nicholson dans Le Pont de la rivière Kwaï (Boulle 1952) et le héros d'Une journée d'Ivan Denissovitch (Soljénitsyne 1962). L'un et l'autre s'absorbent dans leur œuvre -un pont, un mur- au point d'en oublier leur condition de prisonniers. 
62 Il y a d'autres fils conducteurs possible. Nous aurions pu par exemple procéder par genres narratifs (aventures, science-fiction, biographies, essais, romans...) ou par thèmes (la révolte des objets, l'apprenti sorcier...). Mais un simple recensement des thèmes aurait déjà été un travail considérable. Quant aux genres, leur valeur de fil conducteur est assez variable. La science-fiction, par exemple, semble avoir un intérêt évident pour notre propos, d'autant que les histoires de savants fous en font partie. Mais la matière est immense, excessivement hétérogène, et une grande partie relève plutôt du merveilleux le plus traditionnel que de notre domaine. Il existe des anthologies de science-fiction par sous-genres dont certaines sont d'un intérêt plus direct pour nous, comme Des hommes et des machines (Silverberg 1973) ou Histoires de machines (Klein et al. 1974), mais il ne s'en dégage pas de piste évidente. Un autre genre important pour nous est la biographie, y compris l'autobiographie. Car même lorsque le sujet n'est pas homme ou femme de métier, il n'y a pas de biographie qui ne nous parle d'apprentissage, peut-être le thème le plus important de la technologie. Enfin, un genre trop méconnu est celui du comique et de l'humour. J'en ai déjà dit un mot à propos du trickster, des clowns et des bouffons, et de Gribouille sous ses différents déguisements. Je saisis l'occasion pour réparer un oubli impardonnable, celui du baron de Münchhausen ou baron de Crac, dont les aventures sont aussi technologiques que burlesques. Mais plus près de notre époque, nos mésaventures avec le monde des choses et des machines ont inspiré de nombreux auteurs du roman et du cinéma. Dans le roman, les classiques sont Trois hommes dans un bateau et Trois hommes en balade, de Jerome K. Jerome $(1889,1900)$. Dans le cinéma, je pense surtout à certains films de Butser Keaton, mais ce sont tous les classiques qu'il faudrait évoquer, de Mack Sennett à Charlie Chaplin et à W.C. Fields, de Max Linder à Jacques Tati.

On le voit, ce n'est pas la matière qui manque. Les techniques sont là, et bien là, dans les histoires que les hommes se sont toujours plus à se raconter les uns aux autres. Nulle dérobade de la pensée narrative devant un objet qu'elle ne sépare pas des autres, qui ne lui paraît pas plus incongru que les autres et pour lequel elle manifeste souvent une compréhension meilleure que la pensée savante. À tout prendre, par exemple, les histoires de savants fous donnent de l'invention une image moins sommaire que celles dont les philosophes, les sociologues et les économistes se sont longtemps contentés. Et sur les rapports entre technique, désir et plaisir, sujet pratiquement ignoré des sciences sociales, c'est probablement la littérature et le cinéma qui nous en disent le plus.

Les mythes et la fiction sont, pour la Technologie, un domaine immense et à peine exploré. Pour en analyser sérieusement le contenu, il faudrait constituer des corpus documentaires exhaustifs, ou du moins représentatifs, et les analyser en faisant appel à toutes les méthodes nécessaires pour une interprétation pas trop entachée d'anachronisme et d'ethnocentrisme. Nous en sommes loin. À quelques exceptions près, sur l'Antiquité méditerranéenne ou sur Robinson Crusoé, le travail n'a pas même commencé, et ce n'est pas le rapide survol auquel je me suis livré qui peut en tenir lieu. Ce survol ne pouvait pas avoir d'autre but que de reconnaitre le domaine, de le baliser en quelque sorte, pour voir quel pouvait être son intérêt. Sur ce point, chacun se fera sa propre conviction. Je dois avouer que la mienne doit beaucoup, et peut-être trop, au plaisir que j'ai eu à retrouver des histoires qui m'ont enchanté depuis l'enfance, et à en découvrir d'autres qui ont renouvelé cet enchantement. J'avoue même qu'il me suffirait d'avoir fait partager ce plaisir avec quelques lecteurs pour être pleinement satisfait. 


\section{BIBLIOGRAPHIE}

Borchardt, Frank L.

1990. «The Magus as Renaissance Man », The Sixteenth Century Journal 21, 1: 57-76.

Couliano, Ioan P.

1990. « Dr. Faust, Great Sodomite and Necromancer », Revue d'Histoire des Religions 217, 3 : 261-268.

Ducellier, Alain

1976. Le Drame de Byzance. Paris : Hachette.

Eamon, William

1983. « Technology as magic in the late Middle Ages and the Renaissance », Janus 70, 3-4 :171-212.

Frontisi-Ducroux, Françoise

1975. Dédale. Mythologie de l'artisan en Grèce ancienne. Paris : Maspéro.

Ibn Tufayl

1983. Hayy bin Yaqzân. Paris : SPAG [trad. Léon Gauthier].

Klein, Gérard, et al.

1974. Histoires de machines. Paris : LGF (« Livre de Poche »).

Leibniz, Gottfried Wilhelm

1987. « Discours touchant la méthode de la certitude et l'art d'inventer », Techniques \& Culture 9 :

167-174 [Extraits].

Mason, John Hope

1988. « The Character of Creativity : Two Traditions », History of European Ideas 9, $6:$ 697-715.

Michotte, A

1962. Causalité, permanence et réalité phénoménales. Louvain : Publications universitaires; Paris :

Béatrice-Nauwelaerts.

Pellegrin, Pierre

1993. Aristote, La Politique, Livre I. Paris : Nathan.

Pelton, Robert D.

1980. The Trickster in West Africa. Berkeley : University of California Press.

Perrault, Charles

1688. Parallèle des Anciens et des Modernes [...]. Paris : J.-B. Coignard.

Radin, Paul

1956. The Trickster, A Study in American Indian Mythology, with Commentaries (by Karl Kerényi and C.

G. Jung. Londres : Routledge \& Kegan Paul.

Rupp-Eisenreich, Britta

1987. «Culture et technologie : une robinsonade », Techniques \& culture $10: 169-198$.

Sigaut, François

2002. « La formule de Mauss », Techniques \& culture 40 : 153-168.

Silver, Morris

1992. Taking Ancient Mythology Economically. Leyde/New York:

E.J. Brill. 
Silverberg, Robert

1973. Des hommes et des machines. Paris : Verviers, Gérard \& Co (« Bibliothèque Marabout »).

\section{NOTES}

1. Outre les ouvrages littéraires (contes, romans, pièces de théâtre, etc.), qui ne figurent pas dans les références, plusieurs travaux m'ont été très utiles; il s'agit de Borchardt (1990), Couliano (1990), Eamon (1983) Frontisi-Ducroux (1975) et Pelton (1980).

\section{RÉSUMÉS}

Toutes les formes de narration, des plus anciennes aux plus récentes, font une place aux techniques. Car celles-ci font partie des éléments que toute histoire doit emprunter au réel pour trouver des auditeurs ou des spectateurs intéressés. Mais il existe des thèmes littéraires où les techniques interviennent dans la trame même du récit. Certains d'entre eux sont présentés dans cet article : le trickster, Gribouille, Robinson Crusoé, le savant fou, etc. La présence/absence de ces personnages dans l'imaginaire européen suggère que depuis l'Antiquité (Dédale), deux conceptions opposées de l'invention, du travail et de la création y ont alterné.

\section{Techniques in narrative thought.}

All narrative forms, from earliest to newest, treat of techniques. For the latter are part of real world that every story must integrate to interest an audience. But in some literary genres, techniques are involved in the framework of the story. Some of them are presented in this article : the trickster, Gribouille, Robinson Crusoe, the mad scientist, and so on. The presence or the absence of these characters in the european imaginary since Antiquity (Dedale) suggests that two opposite conceptions of invention, work and creation have alternated.

\section{Las técnicas en el pensamiento narrativo.}

Todas las formas de narración, desde las más antiguas hasta las más recientes, dejan un sitio a las técnicas. Pues estas forman parte de los elementos que toda historia tiene que extraer de la realidad para encontrar oyentes o espectadores interesados. Pero existen temas literarios cuya propia trama de relato incluye las técnicas. El artículo presenta algunos de ellos: el trickster, Gribouille, Robinson Crusoe, el científico loco... La pesencia/ausencia de estos personajes en el imaginario europeo sugiere que desde la Antigüedad (Daedalus), alternaron en este último dos concepciones opuestas de la invención, del trabajo y de la creación.

\section{INDEX}

Mots-clés : mythes, magie, invention, savant fou, science-fiction, travail, trickster, Dédale, Faust, Frankenstein, Gribouille, Robinson Crusoé

Keywords : myths, magic, Robinson Crusoe, work 
AUTEUR

FRANÇOIS SIGAUT

EHESS, Centre de Recherches Historiques, 54 Bd Raspail, 75006 Paris 